\title{
Quedas no paciente idoso: o papel do ortopedista na prevenção
}

\author{
Falls among the elderly: the role of orthopedist in the prevention \\ Las caídas en los ancianos: el papel del ortopedista en la prevención \\ Luis Guilherme Rosifini ALVES REZENDE ${ }^{\mathbf{1}}$ \\ Mário Jefferson Quirino LOUZADA ${ }^{2}$ \\ ${ }^{1}$ Residente junto ao Serviço de Ortopedia e Traumatologia do Hospital das Clínicas da \\ Faculdade de Medicina de Ribeirão Preto da Universidade de São Paulo - FMRPUSP \\ ${ }^{2}$ Mestrado em Ciências da Saúde Aplicadas ao Aparelho Locomotor, \\ Departamento de Biomecânica Medicina e Reabilitação do Aparelho Locomotor da FMRPUSP \\ Professor Adjunto, Universidade Estadual Paulista “Júlio de Mesquita Filho” UNESP, Campus de Araçatuba
}

\begin{abstract}
Resumo
O envelhecimento populacional, principal fenômeno demográfico do século 20, trouxe profundas alterações sociais, econômicas e epidemiológicas modificando os padrões de morbidade, invalidez e morte dos indivíduos. As quedas e fraturas representam um dos principais problemas clínicos entre os pacientes idosos. Por sua alta ocorrência, morbimortalidade e elevados custos assistenciais são consideradas importante problema de saúde pública. Cabe ao ortopedista papel ativo na orientação e prevenção de novos episódios das quedas e suas consequências. Realizou-se revisão da literatura obtendo-se artigos na íntegra a partir dos descritores: idoso, acidentes por quedas, fraturas ósseas e ortopedia, publicados até fevereiro de 2015, em periódicos nas bases Lilacs, Bireme e Medline. Utilizou-se roteiro sistematizado de coleta de dados para análise da amostra. Os resultados indicam que a) pelo menos um em cada três idosos apresenta um episódio de queda ao ano com fraturas em até 5\% dos casos; b) as quedas e fraturas impactam fortemente a autonomia e qualidade de vida dos indivíduos idosos; c) as quedas decorrem de etiologia multifatorial, mostrando maior incidência frente a fatores de risco médicos, individuais e domésticos; d) quedas podem ser prevenidas com mudanças no estilo de vida e modificações para maior segurança no ambiente doméstico; e) o ortopedista desempenha importante papel na adoção de hábitos preventivos pelo paciente. Concluiu-se que orientações ao idoso e seus familiares (ou cuidadores) quanto à prevenção de quedas e incentivo ao envelhecimento ativo são medidas eficazes para minimizar a ocorrência de quedas e suas consequências.
\end{abstract}

Descritores: Idoso; Acidentes por Quedas; Fraturas Ósseas; Ortopedia.

\begin{abstract}
Population aging, the main demographic phenomenon of the 20th century, brought changes at social, economic and epidemiological level. These changes modified the patterns of the morbidity, disability and death of individuals. Falls and fractures are a major clinical problem in the elderly people due to the outcomes of these conditions (incidence, health implications, and medical care costs). The orthopedist should take an active role in patient guidance regarding prevention of new episodes of falls and their consequences. The study has conducted a literature review on full articles from descriptors: aged, accidental falls, fractures, bone and orthopedics, published until February 2015, in journals at the basis Lillacs, Bireme and Medline. It was used systematic data collection for sample analysis. The results indicate that a) at least one in three elderly had an episode of fall per year and fractures account for 5\% of cases; b) falls and fractures strongly impact the autonomy and the quality of life of older people; c) the falls result from multifactorial etiology showing higher incidence front to medical risk factor, personal risk factor and risk factor in the home; d) falls can be prevented with healthy lifestyle choices and safety modifications in the home; e) the orthopedist plays an important role in the adoption of preventive habits. It was concluded that guidelines for the elderly people and their families (and caregivers) about the prevention of falls and active aging are efficient measures to minimize the occurrence of falls and their consequences.
\end{abstract}

Descriptors: Aged; Accidental Falls, Fractures, Bone; Orthopedics.

\section{Resumen}

Envejecimiento de la población, el principal fenómeno demográfico del siglo 20, trajo cambios a nivel social, económico y epidemiológico. Estos cambios han cambiado los patrones de la morbilidad, la discapacidad y la muerte de los individuos. Caídas y fracturas son un problema clínico importante en los ancianos debido a los resultados de estas condiciones (incidencia, implicaciones para la salud, y los costos de atención médica). El ortopedista debe asumir un papel activo en la orientación del paciente con respecto a la prevención de nuevos episodios de caídas y sus consecuencias. Se realizó una revisión de la literatura de artículos completos. Los descriptores utilizados fueran: anciano, accidentes por caídas, fracturas óseas y ortopedia. El artículos fueran publicados hasta el mes febrero de 2015 y indexados en las bases: Lillacs, Bireme y Medline. Utilizamos recopilación sistemática de datos para el análisis de la muestra. Los resultados indican que: a) al menos una de cada tres ancianos tuvieron un episodio de caída al año y que las fracturas representan el 5\% de los casos; b) las caídas y fracturas impactan fuertemente la autonomía y la calidad de vida de los ancianos; c) las caídas son el resultado de etiología multifactorial, mostrando mayor incidencia frente a factores de riesgo médicos, personales y domiciliarias; d) caídas se puede prevenir por los cambios en el estilo de vida y modificaciones para una mayor seguridad en el vivenda; e) el ortopedista juega un papel importante en la promoción de hábitos de prevención de las caídas en los ancianos. Se concluyó que las directrices para las personas mayores y sus familias (y cuidadores) sobre la prevención de caídas y importancia del envejecimiento activo son medidas eficaces para minimizar la ocurrencia de caídas y sus consecuencias.

Descriptores: Anciano; Accidentes por Caídas; Fracturas Óseas; Ortopedia. 


\section{INTRODUÇ̃̃O}

O envelhecimento acentuado da população, principal fenômeno demográfico do século 20, resultou do declínio da fecundidade e do aumento da expectativa de vida ${ }^{1}$. Este último, insidioso e lento, é fruto dos melhores índices de desenvolvimento humano e qualidade de vida, sobretudo em razão da expansão do saneamento básico e do advento dos antibióticos e vacinas,2. Em consequência, profundas alterações sociais, econômicas e epidemiológicas modificaram os padrões de morbidade, invalidez e morte dos indivíduos ${ }^{1}$.

No Brasil, indivíduos acima de 60 anos ou mais somam 22,3 milhões ${ }^{3}$, desafiando as ações de promoção de saúde pelos cuidados exigidos, em razão do alto grau de moléstias crônico-degenerativas e de suas complicações, além das disfunções próprias do envelhecimento ${ }^{1}$.

As quedas podem acontecer a qualquer momento e em qualquer lugar para pessoas de qualquer idade ${ }^{4}$. No entanto, as quedas e suas consequências, particularmente as fraturas, constituem um dos principais problemas clínicos da população idosa. Por sua alta incidência, complicações para a saúde, perda da qualidade de vida e altos custos assistenciais, as quedas são consideradas problema de saúde pública ${ }^{5,6}$.

A AAOS (American Academy of Orthopaedic Surgeons $)^{4}$ com base em dados fornecidos pelo CDC (Centers for Disease Control and Prevention) afirma que a cada ano, um em cada três adultos com 65 anos ou mais sofre ao menos uma queda. Em 2000, as quedas entre idosos custaram ao sistema de saúde americano mais de 19 bilhões de dólares, o que equivale a mais de 28,2 bilhões de dólares em 2010.

A SBOT (Sociedade Brasileira de Ortopedia e Traumatologia $)^{7}$ acrescenta que a queda impacta a qualidade de vida, a autonomia e o convívio social do idoso.

A queda pode gerar trauma no idoso levando ao medo de novos episódios, dentro e fora do ambiente doméstico $^{8}$, desencadeando restrição das atividades cotidianas, déficit da capacidade funcional, alterações no equilíbrio/controle postural e baixa autoestima, causando, em muitos pacientes, forte estado depressivo $^{9-11}$

A taxa de mortalidade para quedas aumenta dramaticamente com a idade em ambos os gêneros, em todos os grupos raciais e étnicos.

No lastro dessa discussão, a profilaxia das quedas acena de forma incisiva como medida singular na diminuição da morbidade e mortalidade, gastos hospitalares ou institucionalização do idoso ${ }^{12,13}$.

O perfil profissional do ortopedista o coloca em posição chave na orientação do paciente idoso e seus familiares (ou cuidadores) quanto aos recursos preventivos capazes de minimizar a ocorrência de quedas e suas consequências.

O propósito deste trabalho é promover revisão teórica com ênfase aos fatores de risco para quedas em idosos, alterações decorrentes e importância de medidas preventivas para controle do evento.

\section{MATERIAL E MÉTODO}

Realizou-se revisão da literatura obtendo-se artigos a partir dos descritores: idoso, acidentes por quedas, fraturas ósseas e ortopedia, publicados até fevereiro de 2015, em periódicos nas bases Lilacs, Bireme e Medline. Utilizou-se roteiro sistematizado de coleta de dados para análise da amostra: pesquisa do material, leitura dos artigos e seleção dos textos, totalizando 86 trabalhos. Como critérios de elegibilidade para seleção e inclusão dos artigos na pesquisa por consenso, analisaram-se a procedência da revista e indexação, estudos que apresentassem dados referentes aos descritores selecionados.

\section{MAPA EPIDEMIOLÓGICO}

A queda no indivíduo idoso se dá em decorrência da perda sistemática da estabilidade, graças à insuficiência súbita dos mecanismos neurais e osteoarticulares envolvidos na manutenção do equilíbrio postural ${ }^{14,15}$. O evento não é intencional e leva ao indivíduo a um posicionamento inferior ao que se encontrava, com incapacidade de correção em tempo hábil e apoio no solo ${ }^{16}$.

A queda é uma ocorrência inesperada envolvendo morte, lesão ou risco físico e psicológico, requerendo atenção imediata. Por essa razão pode ser considerada um evento sentinela ${ }^{13}$.

Seu número aumenta progressivamente com a idade em ambos os sexos, em todos os grupos étnicos e raciais. Atinge preferencialmente o gênero feminino ${ }^{17}$ até os 75 anos de idade, quando as frequências passam a se igualar ${ }^{18}$.

Muitos estudos no Brasil buscaram estabelecer, utilizando metodologia diversa, a prevalência de quedas em idosos. Assim, Chaimowicz et al. ${ }^{19}$ descrevem $16.8 \%$ de incidência de queda entre indivíduos com 60 anos ou mais, que faziam uso de 
psicoativos, moradores em um bairro de município mineiro, nos 12 meses que antecederam a coleta de dados. Já Rozenfeld ${ }^{20}$ encontrou índices de $37.4 \%$ entre 634 mulheres que utilizavam algum tipo de medicamento. Ferrer et $\mathrm{al}^{21}$. encontraram em sua amostra de indivíduos com 65 anos ou mais valores de $60 \%$ para a queda. Antes et al. ${ }^{22}$ ouviram 1705 idosos do município de Florianópolis (SC) dentre os quais $18.88 \%$ relataram um episódio de queda nos 11 meses anteriores.

Siqueira et al. ${ }^{23}$ em estudo realizado em 2007 em 41 municípios com mais de 100 mil habitantes em 7 estados brasileiros com 4003 indivíduos com 65 anos ou mais encontrou frequência de $34.8 \%$ significativamente maior nas mulheres $(40.1 \%)$, com fraturas em $12.1 \%$ dos idosos que sofreram queda. Em trabalho posterior realizado em 2011, Siqueira et al. ${ }^{24}$ avaliaram ocorrência de quedas nos 11 meses anteriores à investigação em 6.616 idosos moradores de áreas urbanas de 100 municípios de 23 estados brasileiros e encontraram índices de $27.6 \%$, com fraturas em $11 \%$ dos indivíduos.

Silva et al. ${ }^{25}$ observaram $29 \%$ de prevalência de queda em mulheres com 60 anos ou mais com valores subindo para $51 \%$ naquelas com osteoporose. Estudos realizados por Nascimento et al. $^{26}$ observaram prevalência de $39,1 \%$ de quedas entre idosos, dentre os quais $37,9 \%$ também apresentavam alteração do equilíbrio postural.

A taxa de quedas entre idosos da comunidade, hospitalizados e institucionalizados tende a aumentar nessa ordem. Perracini ${ }^{27}$ lembra que idosos com limitação da autonomia funcional caem 14 vezes mais que os idosos independentes. Estudos de Gabel et al. ${ }^{28}$ apontam que $15 \%$ dos idosos saudáveis caem ao menos uma vez ao ano e este índice sobre para a faixa $30 \%$ a $60 \%$ quando consideramos a população idosa em geral $^{15,29-31}$, o que configura um problema de grandes proporções, principalmente se lembrarmos que esta população está crescendo rapidamente ${ }^{13}$.

Baraff et al. $^{32}$ afirmam que após uma queda a probabilidade de novo episódio nos seis meses seguintes é de dois para três. Pacientes com mais de duas quedas em seis meses devem ser investigados quanto à possibilidade de tratamento das causas ${ }^{13}$.

Cerca de 3 a $5 \%$ dos idosos que sofrem quedas são acometidos por fraturas graves que irão demandar internação, geralmente com o dobro da permanência hospitalar que os idosos admitidos com outro diagnóstico $^{12}$ e apresentando risco de morte de 15 a $50 \%$ no ano seguinte à hospitalização ${ }^{13}$.
Segundo dados do Ministério da Saúde do Brasil, quando consideramos a morbidade, as quedas ocupam o primeiro lugar das internações (56.1\%); além disso, respondem pela terceira posição no óbito por causas externas em pacientes com mais de 60 $\operatorname{anos}^{33}$.

\section{FATORES DE RISCO PARA AS QUEDAS}

Componentes sensoriais, cognitivos (orientação têmporo-espacial; memória; capacidade de cálculo; capacidade de planejamento e decisão; linguagem expressão e compreensão), integrativos centrais (principalmente cerebelo) e musculoesqueléticos ${ }^{13}$ atuam sinergicamente na etiologia das quedas, associados ao efeito cumulativo do fator etário, enfermidades e ambiente desfavorável ${ }^{34-37}$. O risco de queda aumenta em razão direta ao número de fatores $\operatorname{associados}^{16}$.

De um modo geral os fatores de risco para quedas podem ser classificados em intrínsecos, extrínsecos e comportamentais. Os fatores intrínsecos são decorrentes de alterações fisiológicas pelas quais o idoso passa, bem como condições patológicas e efeitos causados por uso de fármacos. Os fatores extrínsecos são dependentes de circunstâncias sociais e ambientais e criam desafios ao idoso. Por sua vez, os fatores comportamentais dizem respeito ao grau de exposição ao risco.

\section{- FATORES INTRÍNSECOS}

A condição clínica do indivíduo idoso é um fator de risco comum patologias cardiovasculares, neurológicas, endocrinológicas, osteomusculares, genito-urinárias, psiquiátricas e sensoriais que comprometam a força muscular, o equilíbrio, a postura e a marcha ${ }^{13,38-40}$.

As doenças crônicas em idosos evoluem de maneira geral com perda da capacidade funcional, e, quando associadas à dependência física e a imobilidade, potencializam a ocorrência de quedas ${ }^{13,41}$.

Também a interação entre queda e déficit sensorial está bem estabelecida ${ }^{13,40}$. Declínio da acuidade visual, redução da percepção da distância, visão periférica e adaptação ao escuro, distúrbios vestibulares e perda da audição impossibilitam ver e ouvir sinais e informações de alarme que poderiam coibir a queda ${ }^{5,33}$.

Carvalho e Coutinho $^{10}$ lembram que o comprometimento da marcha, desequilíbrio, instabilidade postural e aumento do tônus muscular são sintomas que podem sugerir um quadro de demência. Características e deficiências próprias dos quadros de 
demência, tais como o déficit cognitivo, diminuição da massa muscular e densidade óssea, aumentam o risco de episódios de quedas e fraturas entre idosos ${ }^{10,42}$.

A idade avançada mostra associação positiva com o maior numero de quedas em diversos estudos na literatura tais como o de Campbell et al. ${ }^{43}$, Teno et al. $^{44}$, Zang et al. $^{45}$, Chu et al. ${ }^{46}$, Horton e Jones ${ }^{47}$, Gabmann et al. $^{48}$, Ishimoto et al. ${ }^{49}$, Gilbert et al. ${ }^{50}$ e Paranhos $^{51}$. Os efeitos cumulativos das alterações relacionadas à idade explicariam o aumento de queda nos pacientes com o avançar dos anos pelo débito celular e comprometimento dos sistemas responsáveis pelo equilíbrio postural ${ }^{52}$.

Também o uso de medicamentos tem sido relacionado a maior prevalência desses acidentes. Drogas psicoativas ${ }^{53}$, de uso cardiológico (diuréticos, antiarrítmicos, vasodilatadores e glicosídeo cardíaco) e a polifarmácia (uso de quatro ou mais medicamentos simultaneamente $)^{54}$ associam-se ao maior risco de quedas na população acima de 60 anos, especialmente por promoverem sonolência e/ou hipotensão postural $^{9,11,19,35,55-59}$.

O sedentarismo é apontado por vários estudos como importante agente na gênese das quedas em idosos por sua ação tanto nas disfunções músculoesqueléticas $^{13}$, como no processo de envelhecimento, já que alterações fisiológicas e psicológicas observadas no idoso podem ser atribuídas ao estilo de vida sedentário $^{40,60}$.

Santos et al. ${ }^{61}$ assim como Alves e Scheicher ${ }^{62}$ alertam que a institucionalização do idoso representa um fator de risco para episódios de queda, em razão do isolamento social e sedentarismo, com perda progressiva da função e autonomia.

Campbell et al. ${ }^{63}$ apontam maior proporção de mulheres caidoras nas faixas etárias mais avançadas. Igualmente, Berg et al. ${ }^{64}$ apontam neste mesmo grupo maior prevalência de outros fatores intrínsecos que não o gênero: equilíbrio diminuído, marcha lenta, baixa aptidão física, fraqueza muscular, deficiência cognitiva e uso de sedativos e/ou polifarmácia.

Estudos de Pinheiro et al. ${ }^{65}$ com 2420 idosos brasileiros apontaram o medo de cair em em 42,3\% dos homens e $59,8 \%$ das mulheres, especialmente entre os indivíduos com história prévia de quedas e de baixa renda.

O medo de cair geralmente leva à restrição de atividades físicas e sociais. $O$ paciente passa a apresentar alterações comportamentais com pior desempenho da marcha, declínio funcional, isolamento social e diminuição expressiva da qualidade de vida, gerando em muitos casos, dependência física e institucionalização ${ }^{13,66-68}$.

\section{- FATORES EXTRÍNSECOS}

Greg et al. ${ }^{69}$, Fabrício et al. ${ }^{70}$ e Rubenstein ${ }^{71}$ alertam que os fatores extrínsecos responderam por cerca $50 \%$ das quedas entre os idosos avaliados em seus estudos. Esses fatores compreendem eventos ocasionais relacionados ao ambiente que trazem risco aos idosos, especialmente àqueles que já apresentam algum grau de vulnerabilidade, com comprometimento do equilíbrio e marcha. Nesse contexto devem ser consideradas situações que levam o indivíduo a escorregar, tropeçar ou chocar-se contra objetos, pessoas e animais, tais como iluminação insuficiente, superfícies escorregadias, tapetes soltos ou com dobras, degraus altos ou estreitos, obstáculos no caminho (móveis baixos, pequenos objetos, fios), ausência de corrimãos em corredores e banheiros, prateleiras excessivamente baixas ou elevadas, roupas e sapatos inadequados, via pública mal conservada com buracos ou irregularidades e órteses inadequadas ${ }^{69}$.

Rodrigues et al. $^{72}$ avaliaram dados de 102 prontuários de pacientes atendidos em hospital municipal da cidade de São Paulo com diagnóstico de queda. Seus resultados apontaram entre os idosos atendidos por sofrer queda de um nível ao outro, $65 \%$ ocorreram em escada/degrau. Quando avaliados os dados dos pacientes atendidos por sofrer queda do mesmo nível, queda não especificada ou de um nível ao outro, $76,8 \%$ ocorreram na própria residência. As quedas ocorreram no exercício das atividades diárias com $24.8 \%$ de prevalência no período da manhã e $37.6 \%$ no período da tarde. O principal diagnóstico de lesão foi trauma superficial da cabeça $(26,6 \%)$, seguido por ferimento da cabeça $(6,4 \%)$ e trauma superficial do punho e da mão $(5,5 \%)$. Os pacientes evoluíram para alta hospitalar em $72.5 \%$ dos casos. $4,6 \%$ necessitaram de acompanhamento; $1,8 \%$ internação e 3,7\% ficaram sob observação, após o acidente.

Em seus estudos Fabrício et al. $^{70}$ encontraram maior prevalência de quedas em situações de piso escorregadio (26\%), atrapalhar-se com objetos no chão (22\%), colisão com outras pessoas $(11 \%)$, subir em objetos para alcançar algo (7\%), queda da cama $(7 \%)$ e problemas com degrau (7\%).

Rocha et al. ${ }^{73}$ apontam como fatores ambientais críticos desnível na soleira das portas, tapetes soltos, escadas sem corrimão, objetos guardados em locais altos, piso escorregadio (especialmente em banheiros), vestuário/calçados inadequados, cadeiras/camas muito 
altas ou muito baixas, móveis instáveis e deslizantes e iluminação deficiente.

Marin et al. ${ }^{74}$ avaliaram os fatores relacionados ao risco de queda em 51 idosos residentes em microárea e observaram em média dois fatores de risco para cada idoso investigado, com maior prevalência para domicílio com banheiro sem piso antiderrapante $(60,7 \%)$, tapetes e objetos soltos pela casa (49\%) e piso escorregadio $(45, \%)$.

Pinho et al. ${ }^{75}$ alertam que idosos saudáveis tendem a cair durante atividades instrumentais e em ambientes públicos, enquanto idosos frágeis tendem a cair em atividades rotineiras nos seus próprios domicílios, sem grandes exigências de equilíbrio.

Pinho et al. ${ }^{75}$ também observaram que $36 \%$ das quedas se deviam a problemas no ambiente, demonstrando a necessidade da adequação da moradia. Também Fabrício et al. $^{70}$ estudaram aa causas e consequências de quedas de 50 idosos, de ambos os sexos, com idade de 60 anos ou mais, residentes em Ribeirão Preto, SP, que haviam sido atendidos em duas unidades de um hospital público. Foram consultados prontuários e realizadas visitas domiciliares para aplicação de questionário estruturado com perguntas abertas, fechadas e mistas relativas à queda. Seus resultados mostraram que $54 \%$ das quedas eram decorrentes de ambiente físico inadequado.

Lopes et al. ${ }^{76}$ observaram em seus estudos que as quedas ocorreram predominantemente, em ambientes como banheiro e cozinha. Predomínio de quedas perto do domicílio, no quintal, dormitório, banheiro e cozinha são achados consistentes com os estudos de Pinho et al. ${ }^{75}$, Tinetti ${ }^{77}$ e Vassalo et al. ${ }^{78}$.

Carter et al. ${ }^{79}$ investigaram episódios de quedas em 425 idosos e observaram que os locais que ocorreram maior número de acidentes foram ao redor do domicílio, cozinha e dormitório.

\section{- FATORES COMPORTAMENTAIS}

Os fatores comportamentais dizem respeito ao grau de exposição ao risco de fratura pelo idoso. $\mathrm{O}$ maior risco de fratura incide tanto sobre as pessoas mais inativas como também sobre as mais ativas, pela maior fragilidade do primeiro grupo e maior exposição ao risco no segundo grupo.

Segundo Messias e Neves ${ }^{80}$ algumas atividades que resultam em quedas decorrem de comportamentos imprudentes, a exemplo de subir uma escada dobrável para limpar o topo de um armário.

Estudo de Ferrer et al. ${ }^{21}$ revelou alta prevalência de comportamentos de risco entre idosos tais como adentrar ambiente escuro, não utilizar iluminação noturna e subir em banco para alcançar objetos.
Ribeiro et al. $^{81}$ acrescentam o uso de calçados inadequados, relatado por $64,7 \%$ da população idosa.

Messias e $\mathrm{Neves}^{80}$ destacam que, quando se avaliam idosos que nunca caíram e que apresentam bom estado funcional, tão importante quanto a presença ou a exposição ao risco ambiental são as atitudes de risco.

\section{PREVENÇÃO DAS QUEDAS}

Projeta-se para 2050 um percentual de $19 \%$ e $28 \%$, respectivamente para os indivíduos com 65 ou 80 anos ou mais, alterando drasticamente a pirâmide demográfica brasileira. Além disso, a população mais idosa será eminentemente feminina, com 76 idosos para cada 100 idosas. Acima de 80 anos serão duas mulheres para cada homem ${ }^{1}$. O perfil epidemiológico também sofrerá alterações com maior incidência de doenças cuja idade seja o principal fator de risco, além do aumento da prevalência de doenças crônicas, incrementando a polifarmácia ${ }^{1}$, e da prevalência de quedas.

As quedas impactam fortemente o cotidiano do paciente idoso. Programas de prevenção das quedas e incentivo ao envelhecimento ativo contribuem para a manutenção da autonomia e diminuição significativa da morbidade, custos assistenciais hospitalares, possibilidade de institucionalização e mortalidade. Paciente e seus familiares devem ser orientados quanto ao benefício gerado pelo acompanhamento médico regular, dieta equilibrada, prática de atividade física e convívio social ativo ${ }^{13}$. Devem ser identificados por meio de anamnse criteriosa os fatores de risco intrínsecos e extrínsecos, bem como deve ser levantado história prévia de quedas ${ }^{13,82}$. Ganança et al. ${ }^{82}$ lembram que a identificação de fatores de risco para quedas que possam ser modificados por meio de intervenções específicas é essencial, tanto na prevenção de futuros episódios, como no manejo do processo de reabilitação.

Além do exame físico tradicional é indicada a realização do teste get-up and go (levante-se e ande), o qual avalia sobretudo a marcha. Para sua realização, o paciente sentado em uma cadeira sem braços deverá levantar-se e caminhar cerca de três metros até uma parede, virar-se sem tocá-la, retornar à cadeira e sentarse novamente. O exercício deverá ser repetido com rotação em sentido oposto. Deverá ser observado eventual desequilíbrio na rotação do corpo, simetria e firmeza dos passos, bem como a facilidade em se sentar e levantar ${ }^{13,83}$. A cotação pode oscilar entre 1 (sem qualquer sinal de instabilidade) e 5 (onde há um risco permanente de queda $)^{83}$. Também a Prova de 
Tinetti pode ser aplicada para avaliação do equilíbrio estático (normal, adaptado e anormal) e dinâmico (normal e anormal) $^{83}$.

Também a qualidade de vida do paciente idoso deve ser investigada por meio de instrumento validado tal como Dizziness Handicap Inventory (DHI) que avalia múltiplos aspectos da vida diária e a sua correlação com os distúrbios do equilíbrio, permitindo avaliar a importância relativa das dimensões física, funcional e emocional do desequilíbrio ${ }^{83}$.

Esses dados contribuem para medidas preventivas e abordagem múltipla e integral direcionada às causas da queda em idosos. O ideal é adiar a inaptidão, manter as habilidades físicas e mentais, para uma vida independente e autônoma ${ }^{5}$.

Otimização medicamentosa ${ }^{13}$, reabilitação de distúrbios visuais, de equilíbrio, marcha e força ${ }^{83}$, implementação de atividades físicas programadas e correção dos fatores de risco ambientais são intervenções eficazes na retomada das atividades cotidianas, minimizando possíveis efeitos emocionais e psicológicos, especialmente no paciente com histórico de queda anterior ${ }^{13,82,84}$.

A prevenção de quedas necessariamente envolve mudança de paradigma nos modelos de promoção de saúde, arquitetura e urbanização, com o propósito de orientar e preparar a sociedade para as limitações morfológicas, funcionais e bioquímicas que acompanham o processo de envelhecimento, as quais tornam o organismo susceptível às agressões intrínsecas e extrínsecas ${ }^{85}$.

Frente a essa necessidade, a Sociedade Brasileira de Ortopedia (SBOT) implantou em 1999, durante o ano internacional do idoso, com apoio do Ministério da Saúde, instituições e profissionais da arquitetura e da construção civil, o Projeto Casa Segura trazendo o conceito de moradia, com ambientes equipados com itens de segurança que proporcionem ao idoso não só segurança e conforto, mas também independência, autonomia, dignidade e qualidade de vida ${ }^{86}$.

Cabe assim ao ortopedista, adoção de medidas preventivas em relação às quedas, por meio da elaboração de um plano de intervenção a curto, médio e longo prazo, visando atingir objetivos específicos para cada paciente, em comum acordo com o próprio idoso, seus familiares e/ou cuidadores. Esses objetivos devem ser estrategicamente estabelecidos em função dos fatores de risco, individuais para cada idoso, e periodicamente devem ser reavaliados, a fim de se manterem atitudes, hábitos e comportamentos seguros em suas atividades e no meio em que vivem.

\section{CONCLUSÃO}

Orientar o idoso, seus familiares e/ou cuidadores sobre os riscos de queda e suas consequências contribui para redução das ocorrências e instalação de situações de limitação e/ou dependência.

A queda é um evento real na vida do paciente. Sua gravidade potencial confere às medidas preventivas garantia de autonomia, capacidade funcional e independência ao idoso. Para tanto, a identificação integral dos fatores de risco que possam ser modificados por meio de propostas e intervenções pontuais e específicas faz-se importante.

\section{REFERÊNCIAS}

1 Nasri F. O envelhecimento populacional no Brasil. Einstein. 2008; 6 (Supl 1):S4-S6

2 Ramos LR, Veras R, Kalache A. A populational aging: a brazilian reality. Rev Saúde Publica. 1987;21(3):211-24.

3 Instituto Brasileiro de Geografia e Estatística. Disponível em: http://www.ibge.gov.br/home/ estatistica/populacao/ censo2010/default.shtm. Acesso em: 10 jan. 2015

4 American Academy of Orthopaedic Surgeons. Guidelines for Preventing Falls - OrthoInfo AAOS. Disponível em: http://orthoinfo.aaos.org/ topic.cfm? topic=a00135. Acesso em: 15 jan. 2015.

5 Almeida LP, Brites MF, Takizawa MGMH. Quedas em idosos: fatores de risco. RBCEH. 2011;8(3):384-91.

6 Ricci NA, Gonçalves DFF, Coimbra IB, Coimbra AMV. Fatores associados ao histórico de quedas de idosos assistidos pelo programa de saúde da família. Saude Soc. 2010; 19(4):898-909.

7 Sociedade Brasileira de Ortopedia e Traumatologia. Quedas em idosos: a prevenção é o melhor tratamento/SBOT. Disponível em: http://www.sbot.org.br/ blogsbot/ index.php /archives/ quedas-em-idosos-a-prevencao-e-omelhor-tratamento. Acesso em: 12 jan.2015.

8 Carvalho EMR, Garcês JR, Menezes RL, Silva EFC. O olhar e o sentir do idoso no pós-queda. Rev bras geriatr gerontol. 2010;13(1):7-16.

9 Hamra A, Ribeiro MB, Miguel OF. Correlação entre fratura por queda em idosos e uso prévio de medicamentos. Acta ortop bras. 2007;15(3): 143-5.

10 Carvalho AM, Coutinho ESF. Demência como fator de risco para fraturas graves em idosos. Rev Saúde Pública. 2002;36(4):448-54.

11 ESF Coutinho, SD Silva. Uso de medicamentos 
como fator de risco para fratura grave decorrente de queda em idosos. Cad. Saúde Pública. 2002; 18(5):1359-66.

12 Fuller GF. Falls in the elderly. Am Fam Physician. 2000;61(7):2159-68.

13 Sociedade Brasileira De Geriatria e Gerontologia. Quedas em idosos: prevenção. Projeto Diretrizes AMB e CFM. 2008. Disponível em: http://www. projetodiretrizes. org.br/ projeto_diretrizes/ 082.pdf. Acesso em: 13 jan. 2015.

14 Cunha UGV, Guimarães RM. Sinais e sintomas do aparelho locomotor. In: Guimarães RM, Cunha UGV. (Org.). Sinais e sintomas em geriatria. Rio de Janeiro: Revinter, 1989.

15 Perracini MR, Ramos LR. Fatores associados a quedas em uma coorte de idosos residentes na comunidade. Rev Saúde Pública. 2002;36(6):70916.

16 Nevitt MC. Falls in the elderly: risk factors and prevention. In: Masdeu,JC, Sudarsky L, Wolfson L (edit.). Gait disorders of aging. Falls and Therapeutic strategies. Philadelphia: LippincottRaven Publishers, 1997.

17 Graziano KU, Maia FOM. Principais acidentes de causa externa no idoso. Rev Gerontologia. 1999; 7:133-9.

18 Abreu DROM. Fatores associados à recorrência de quedas de idosos na comunidade. [dissertação], Cuiabá: Faculdade de Enfermagem, Universidade Federal do Mato Grosso; 2013.

19 Chaimowicz F, Ferreira TJXM, Miguel DFA. Use of psychoactive drugs and falls among older people living in a community in Brazil. Rev Saúde Pública. 2000;34(6):631-635.

20 Rozenfeld S. Reações Adversas aos Medicamentos na Terceira Idade: As Quedas como Iatrogenia Farmacoterapêutica. [tese], Rio de Janeiro: Instituto de Medicina Social, Universidade do Estado do Rio de Janeiro; 1997.

21 Ferrer MLP, Perracini MR, Ramos LR. Prevalência de fatores ambientais associados a quedas em idosos residentes na comunidade em São Paulo, SP. Rev Bras Fisioter. 2004;8(2):149-54.

22 Antes DL, Schneider IJC, Benedetti TRB, d'Orsi E. Medo de queda recorrente e fatores associados em idosos de Florianópolis, Santa Catarina, Brasil. Cad. Saúde Pública. 2013;29(4): 758-68.

23 Siqueira FV, Facchini LA, piccini RX, Tomasi E, Thumé E, Silveira DS, et al. Prevalência de quedas em idosos e fatores associados. Rev Saúde Pública. 2007;41(5):749-56
24 Siqueira FV, Facchini LA, Silveira DS, Piccini RX, Tomasi E, Thumé E, et al. Prevalence of falls in elderly in Brazil: a countrywide analysis. Cad Saúde Pública. 2011; 27(9): 1829-36.

25 Silva RS, Costa Paiva L, Oshima MM, Morais SS, Pinto Neto AM. Frequência de quedas e associação com parâmetros estabilométricos de equilíbrio em mulheres na pós-menopausa com e sem osteoporose. Rev Bras Ginecol Obstet. 2009;31(10):496-502.

26 Nascimento BN, Duarte BV, Antonini DG, Borges SM. Risco para quedas em idosos da comunidade: relação entre tendência referida e susceptibilidade para quedas com o uso do teste clínico de interação sensorial e equilíbrio. Rev Soc Bras Clin Méd. 2009;7(2):95-9.

27 Perracini MR. Fatores associados a quedas em uma coorte de idosos residentes no município de São Paulo [tese]. São Paulo: Escola Paulista de Medicina, Universidade Federal de São Paulo; 2000.

28 Gabell A, Simons MA, Nayak US. Falls in the healthy elderly: predisposing causes. Ergonomics.1985;28(7):965-75.

29 Ryynänen OP, Kivelä SL, Honkanen R, Laippala $\mathrm{P}$, Soini P. Incidence of falling injuries leading to medical treatment in the elderly. Public Health. 1991;105(5):373-86.

30 Sattin RW. Falls among older persons: A public health perspective. Annu Rev Public Health.1992;13:489-508.

31 Tinetti ME. Prevention of falls and fall injuries in elderly persons: a research agenda. Prev Med. 1994;23(5):756-62.

32 Baraff LJ, Della Penna R, Willians N, Sanders A. Practice guideline for the ED Management of falls in community - dwelling elderly persons. Ann Emerg Med. 1997;30:480-92.

33 Gawryszewski VP, Jorge MHPM, Koizumi MS. Mortes e internações por causas externas entre os idosos no Brasil: o desafio de integrar a saúde coletiva e a atenção individual. Rev Assoc Med Bras. 2004;50(1):97-103.

34 Tallis R, Fillit SHM, Brocklehurst TJC, eds. Brocklehurst's textbook of geriatric medicine and gerontology. London: Churchill Livingstone, 1998.

35 Tinetti ME, Speechley M, Ginter SF. Risk factors for falls among elderly persons living in the community. N Engl J Med. 1988;319(26):1701-7.

36 Campbell AJ, Borrie MJ, Spears GF, Jackson SL, 
Brown JS, Fitzgerald JL. Circumstances and consequences of falls experienced by a community population 79 years and over during a prospective study. Age Ageing. 1990;19(2):136-41.

37 Reyes-Ortiz CA, Al Snih S, Markides KS. Falls among elderly persons in Latin America and the Caribbean and among elderly Mexican--Americas. Rev Panam Salud Publica. 2005;17(5-6):362-9.

38 Costa ICP, Lopes MEL, Andrade CG, Souto MC, Costa KC, Zaccara AAL. Fatores de Risco de Quedas em Idosos: Produção Científica em Periódicos Online no Âmbito da Saúde. R bras ci Saúde. 2012;16(3):445-52.

39 Cunha AA, Lourenço RA. Quedas em idosos: prevalência e fatores associados. Revista Hospital Universitário Pedro Ernesto. 2014;13(2):21-9.

40 Kay PD, Tideiksaar R. Quedas e distúrbios de marcha. In: Abrams WB, Berkow R. Manual Merck de Geriatria. São Paulo: Ed Roca; 1995.

41 Celich KLS, Souza SMS, Zenevicz L, Orso ZA. Fatores que predispõem às quedas em idosos. RBCEH. 2010;7(3):419-26.

42 Berlinger WG, Potter JF. Low Body Mass Index in demented outpatients. J Am Geriatr Soc. 1991;39(10):973-8.

43 Campbell AJ, Borrie MJ, Spears GF. Risk factors for falls in a community-based prospective study of people 70 years and older. J Gerontol. 1989;44(4):M112-7.

44 Teno J, Kiel DP, Mor V. Multiple stumbles: a risk factor for falls in community-dwelling elderly: a prospective study. J Am Geriatr Soc. 1990;38(12):1321-25.

45 Zhang JG, Ishikawa-Takata K, Yamazaki H, Ohta T. Is a type A behavior pattern associated with falling among the community-dwelling elderly? Arch Gerontol Geriatr. 2004;38(2):145-52.

46 Chu LW, Chi I, Chiu AY. Incidence and predictors of falls in the Chinese elderly. Ann Acad Med Singapore. 2005;34(1):60-72.

47 Horton JC, Jones MR. Warning on inaccurate Rosenbaum cards for testing near vision. Surv Ophthalmol. 1997;42(2):169-74.

48 Gabmann KG, Rupprecht R, Freiberger E, IZG Study Group. Predictors for occasional and recurrent falls in community-dwelling older people. Gerontol. Geriat. 2009;42(1): 3-10.

49 Ishimoto $\mathrm{Y}$, Wada T, Hirosaki M, Kasahara Y, Kimura Y, Konno A, et al. Age and sex significantly influence fall risk in communitydwelling elderly people in Japan. J Am Geriatr Soc. 2009;57(5):930-2.
50 Gilbert T, Szczerbinska K, Ziere G, Ait S, Bonnefoy M, van der Velde N, et al. Management of falls: France, the Netherlands, Poland. European Geriatric Medicine. 2012;3(4):246-53.

51 Paranhos WY. Trauma no idoso. In: Sousa RMC, Calil AM, Paranhos WY, Malvestio MA. Atuação no trauma: uma abordagem para a enefermagem. São Paulo: Atheneu, 2009.

52 Beck AP, Antes DL, Meurer ST, Benedetti TRB, Lopes MA. Fatores associados às quedas entre idosos praticantes de atividades físicas. Texto Contexto Enferm. 2011;20(2):280-6.

53 Campbell AJ, Robertson MC, Gardner MM, Norton RN, Buchner DM. Psychotropic medication withdrawal and a home-based exercise program to prevent falls: a randomized, controlled trial. J Am Geriatr Soc. 1999;47(7):850-3.

54 Bueno-Cavanillas A, Padilla-Ruiz F, JiménezMoleón JJ, Peinado-Alonso CA, Galvez-Vargas R. Risk factors in falls among the elderly according to extrinsic and intrinsic precipitating causes. Eur $\mathrm{J}$ Epidemiol. 2000;16(9):849-59.

55 Leipzig RM, Cumming RG, Tinetti ME. Drugs and falls in older people: A systematic review and meta-analysis: I. Psychotropic drugs. J Am Geriatr Soc. 1999;47(1):30-9.

56 Leipzig RM, Cumming RG, Tinetti ME. Drugs and falls in older people: A systematic review and meta-analysis: II. Cardiac and analgesic drugs. J Am Geriatr Soc. 1999;47(1):40-9.

57 Cumming RG, Miller JP, Kelsey JL, Davis P, Arfken CL, Birge SJ, et al. Medications and multiple falls in elderly people: The St. Louis OASIS Study. Age Ageing. 1991; 20(6):455-61.

58 Monane M, Avorn J. Medications and falls. Causation, correlation, and prevention. Clin Geriatr Med. 1996; 12(4):847-58.

59 Rozenfeld S, Camacho LA, Veras P. Medication as a risk factor for falls in older women in Brazil. Rev Panam Salud Publica. 2003;13(6):369-75.

60 Tinetti ME, Inouye SK, Gill TM, Doucette JT. Shared risk factors for falls, incontinence, and functional dependence. Unifying the approach to geriatric syndromes.JAMA. 1995;273(17):1348-53.

61 Santos MLC, Andrade MC. Incidência de quedas relacionadas aos fatores de riscos em idosos institucionalizados. Rev Baiana de Saúde Pública. 2005;29(1):57-68.

62 Alves NB, Scheicher ME. Equilíbrio postural e risco para queda em idosos da cidade de Garça, SP. Rev Bras Geriatr Gerontol. 2011;14(4):763-8. 
63 Campbell AJ, Spears GF, Borrie MJ. Examination by logistic regression modelling of the variables which increase the relative risk of elderly women falling compared to elderly men. J Clin Epidemiol. 1990;43(12):1415-20.

64 Berg WP, Alessio HM, Mills EM, Tong C. Circunstances and consequences of falls in independent community - dwelling older adults. Age Ageing. 1997; 26(4):261-8, 1997.

65 Pinheiro Mde M, Ciconelli RM, Martini LA, Ferraz MB. Risk factors for recurrent falls among Brazilian women and men: the Brazilian Osteoporosis Study. Cad Saude Publica. 2010;26(1):89-96.

66 Pereira CLN, Vogelaere P, Baptista F. Role of physical activity in the prevention of falls and their consequences in the elderly. Eur Rev Aging Phys Act. 2008;5(1):51-8.

67 Montero-Odasso M. Falls as a geriatric syndrome: how to prevent them? How to treat them? In: Duque G. Kiel DP. Osteoporosis in older person. Springer-verlag: London, 2009.

68 Mahant PR, Stacy MA. Movement disorders and normal aging. Neurol Clin. 2001;19(3):553-63.

69 Gregg EW, Pereira MA, Caspersen CJ. Physical activity, falls and fracturesamong older adults: a review of the epidemiologic evidence. J Am Geriatr Soc. 2000;48(8):883-93.

70 Fabrício SCC, Rodrigues RAP, Costa Junior ML. Causas e consequiências de quedas de idosos atendidos em hospital público. Rev Saude Pública. 2004;38(1):93-9.

71 Rubenstein LZ. Falls in older people: epidemiology, risk factors and strategies for prevention. Age Ageing. 2006;35(Suppl 2):ii37ii41.

72 Rodrigues CL, Fernandes A, Armond JE, Gorios C. Fatores associados à queda em idosos atendidos em hospital da região sul da cidade de São Paulo. Rev Bras Ciências da Saúde. 2013;36(2):14-9.

73 Rocha L, Budó MLD, Beuter M, Silva RM, Tavares JP. Vulnerabilidade de idosos às quedas seguidas de fratura de quadril. Esc Anna Nery. 2010;14(4):690-6.

74 Marin MJS, Amaral FS, Martins IB. Identificando os fatores relacionados ao diagnóstico de enfermagem "risco de quedas" entre idosos. Rev bras enferm. 2004;57(5):560-4.

75 Pinho TAM, Silva AO, Tura LFR, Moreira MASP, Gurgel SN,Smith AAF, et al. Avaliação do risco de quedas em idosos atendidos em Unidade Básica de Saúde. Rev Esc Enferm USP; 2012, 46(2):320-7.
76 Lopes MCL, Violin MR, Lavagnoli AP, Marcon SS. Fatores desencadeantes de quedas no domicílio em uma comunidade de idosos. Cogitare Enferm. 2007;12(4):472-7.

77 Tinetti ME. Clinical practice: preventing falls in elderly persons. N Engl J Med. 2003;348(1):42-9.

78 Vassalo M, Stockdale R, Sharma JC, Briggs R, Allen S. A comparative study of the use of four falls risk assessment tools on acute medical wards. J Am Geriatr Soc. 2005;53(6):1034-8.

79 Carter SE, Campbell EM, Sanson-Fisher RW, Gillesple WJ. Accidents in older people living at home: a community based study assessing prevalence, type, location and injuries. Austr NZJ. Public Health. 2000;24(6):633-6.

80 Messias MG, Neves RF. A influência de fatores comportamentais e ambientais domésticos nas quedas em idosos. Rev bras geriatr gerontol. 2009; 12(2):275-82.

81 Ribeiro AP, Souza ER, Atie S, Souza AC, Schilithz AO. A influência das quedas na qualidade de vida de idosos. Ciênc. saúde coletiva. 2004;13(4):126573.

82 Ganança FS, Gazzola JM, Aratani MC, Perracini MR, Ganança MM. Circunstâncias e consequências de quedas em idosos com vestibulopatia crônica. Rev Bras Otorrinolaringol. 2006;72(3):388-93.

83 Luzio CS, Garcia FV, Benzinho T, Veiga VG. Programa de prevenção de quedas em idosos. Disponível em: ww.otoneuro.pt/index.php /artigos/artigos-para-discussao/113-programa-deprevencao-de-quedas-no-idoso. Acesso em: 15 Jan.2015.

84 Motta LB, Aguiar A, Coutinho ESF, Huf G. et al. Prevalência e fatores associados a quedas em idosos de um município do Rio de Janeiro. Rev bras geriatr gerontol. 2010;13(1):83-91.

85 Mantello EB, Moriguti JC, Rodrigues Júnior AL, Ferrioli E. Efeito da reabilitação vestibular sobre a qualidade de vida de idosos labirintopatas. Rev Bras Otorrinolaringol. 2008;74(2):172-80.

86 Sociedade Brasileira de Ortopedia (SBOT). Disponível em: http://www.portalsbot.org.br/wpcontent/ uploads/ 2014/11/Jornal-da-SBOT-n.41junho-2003.pdf. Acesso em: 15 Jan.2015. 


\section{CONFLITO DE INTERESSES}

Os autores declaram não haver conflitos de interesse.

\section{AUTOR PARA CORRESPONDÊNCIA}

Luis Guilherme Rosifini Alves Rezende

lugui.rezende@ hotmail.com

Submetido em 11/03/2015

Aceito em 16/03/2015 